\title{
Validation of whole-blood transcriptome signature during microdose recombinant human erythropoietin (rHuEpo) administration
}

Guan Wang ${ }^{1,10}$, Jérôme Durussel ${ }^{2}$, Jonathan Shurlock³ ${ }^{3}$ Martin Mooses ${ }^{4}$, Noriyuki Fuku' ${ }^{5}$, Georgie Bruinvels ${ }^{6}$, Charles Pedlar ${ }^{6}$, Richard Burden ${ }^{6}$, Andrew Murray ${ }^{7}$, Brendan Yee ${ }^{8}$, Anne Keenan², John D. McClure ${ }^{2}$,

Pierre-Edouard Sottas ${ }^{9}$ and Yannis P. Pitsiladis ${ }^{1,10^{*}}$

From 34th FIMS World Sports Medicine Congress

Ljubljana, Slovenia. $29^{\text {th }}$ September $-2^{\text {nd }}$ October 2016

\begin{abstract}
Background: Recombinant human erythropoietin (rHuEpo) can improve human performance and is therefore frequently abused by athletes. As a result, the World Anti-Doping Agency (WADA) introduced the Athlete Biological Passport (ABP) as an indirect method to detect blood doping. Despite this progress, challenges remain to detect blood manipulations such as the use of microdoses of rHuEpo.

Methods: Forty-five whole-blood transcriptional markers of rHuEpo previously derived from a high-dose rHuEpo administration trial were used to assess whether microdoses of rHuEpo could be detected in 14 trained subjects and whether these markers may be confounded by exercise ( $n=14$ trained subjects) and altitude training ( $n=21$ elite runners and $n=4$ elite rowers, respectively). Differential gene expression analysis was carried out following normalisation and significance declared following application of a $5 \%$ false discovery rate (FDR) and a 1.5 foldchange. Adaptive model analysis was also applied to incorporate these markers for the detection of rHuEpo.

Results: ALAS2, BCL2L1, DCAF12, EPB42, GMPR, SELENBP1, SLC4A1, TMOD1 and TRIM58 were differentially expressed during and throughout the post phase of microdose rHuEpo administration. The CD247 and TRIM58 genes were significantly up- and down-regulated, respectively, immediately following exercise when compared with the baseline both before and after rHuEpo/placebo. No significant gene expression changes were found 30 min after exercise in either rHuEpo or placebo groups. ALAS2, BCL2L1, DCAF12, SLC4A1, TMOD1 and TRIM58 tended to be significantly expressed in the elite runners ten days after arriving at altitude and one week after returning from altitude (FDR $>0.059$, fold-change varying from 1.39 to 1.63). Following application of the adaptive model, 15 genes showed a high sensitivity ( $\geq 93 \%$ ) and specificity ( $\geq 71 \%)$, with BCL2L1 and CSDA having the highest sensitivity $(93 \%)$ and specificity $(93 \%)$.

(Continued on next page)
\end{abstract}

\footnotetext{
* Correspondence: y.pitsiladis@brighton.ac.uk

${ }^{1}$ Centre of Sports Medicine for Anti-Doping Research, University of Brighton, Eastbourne, UK

${ }^{10}$ Department of Movement, Human and Health Sciences, University of

Rome "Foro Italico", Rome, Italy

Full list of author information is available at the end of the article
} 
(Continued from previous page)

Conclusions: Current results provide further evidence that transcriptional biomarkers can strengthen the ABP approach by significantly prolonging the detection window and improving the sensitivity and specificity of blood doping detection. Further studies are required to confirm, and if necessary, integrate the confounding effects of altitude training on blood doping.

Keywords: Recombinant human erythropoietin, Whole blood, Transcriptome, Altitude, Exercise, Athlete biological passport

\section{Background}

The performance-enhancing drug recombinant human erythropoietin (rHuEpo) stimulates red blood cell production and although the World Anti-Doping Agency (WADA) prohibits its use, is frequently abused by athletes. The early anti-doping approach was to set an upper limit for haemoglobin and haematocrit levels in an attempt to discover rHuEpo abuse [1]. The first direct analytical procedure to detect rHuEpo was introduced in 2000 and exploited the differences in the charge profiling of endogenously and exogenously produced Epo in urine by isoeletric focusing (IEF) [2]. The main limitations of this direct approach are a variable and short detection window and low sensitivity [3]. There is now an improved direct analytical test to detect rHuEpo using sarcosyl polyacrylamide gel electrophoresis (SAR-PAGE, a modified sodium dodecyl sulfate polyacrylamide gel electrophoresis) with increased discriminatory capacity compared to IEF and detection window of 24 to $85 \mathrm{~h}$ using blood and urine samples [4]. According to the WADA technical document (i.e. TD2014EPO), SARPAGE is currently recommended for rHuEpo detection in both the initial and confirmation testing procedures [5]. Despite these advances, important limitations in detection of the direct approach have prompted a paradigm shift to the indirect identification of the effect of the prohibited method and/or substance.

One of the initial indirect methods involved the use of altered haematological markers such as reticulocyte haematocrit, haematocrit and percent macrocytes for the detection of current and recent rHuEpo intake using the ON- and OFF-statistical models, respectively [6]. This further advance culminated in the development of the Athlete Biological Passport (ABP) [1], introduced in 2009 by WADA. The ABP monitors changes in the blood matrix of an individual over time using Bayesian inference techniques to establish an individual's haematological profile that can reveal evidence of doping, not confined only to rHuEpo but also other forms of blood manipulation [7]. The stability of the ABP haematological parameters is limited to $48 \mathrm{~h}$ for reticulocytes and $72 \mathrm{~h}$ for haemoglobin from blood collection to analysis when samples are handled at $4{ }^{\circ} \mathrm{C}$ and a limit of $36 \mathrm{~h}$ has been recommended by WADA for improved analytical quality [8]. A particular advantage of the ABP approach is the incorporation of other evidence of doping, such as longitudinal performance data, additional biomarkers yet to be discovered and validated and other nonanalytical evidence [1]. Despite this significant advance, the detection of rHuEpo and blood doping in general, using the current $\mathrm{ABP}$ approach, remains unsatisfactory $[9,10]$. For example, the application of the ABP failed to reveal any evidence of rHuEpo microdosing (i.e. 20-30 IU. $\mathrm{kg}^{-1}$ body mass rHuEpo twice weekly for 8 weeks) in 10 healthy male subjects [11]. In addition, the haematological parameters of the ABP may be confounded with factors such as altitude exposure, since hypoxia may affect the blood variables and vascular volumes $[12,13]$. Notwithstanding these limitations, the addition of other biomarkers to the ABP is envisaged to improve the sensitivity and specificity of the ABP model, and therefore substantially enhancing future doping detection strategies $[1,14,15]$.

Current advances in omics technologies permit a global transcriptional, translational or epigenomic feature of a cell, tissue or organism under altered physiological or developmental conditions to be investigated. For example, a state-of-the-art omics approach has been successfully applied to epidemic diseases (e.g. [16, 17]) and cancer diagnosis [18]. Undoubtedly, the application of omics to the field of anti-doping will help reveal potential doping biomarkers. In a study by Varlet-Marie et al., the gene expression profile in response to darbepoetin alpha was determined using the serial analysis of gene expression (SAGE) by pooling whole-blood samples from 14 healthy, active subjects (50\% male) into three SAGE libraries (before, during and after administration) [19]. The authors then confirmed the differential expression of 95 genes identified using SAGE in two well-trained male athletes by qPCR [19]. Five genes remained significantly expressed following a further high dose and microdose rHuEpo treatment in these athletes based on a fold change of 1.5 and a false discovery rate (FDR) of 0\% [19]. This initial promise of improved 
discriminatory potential of the transcriptomic biomarkers to detecting doping, encouraged a number of other attempts to investigate the global gene expression patterns in whole blood or lymphocytes for the detection of testosterone, anabolic steroids, recombinant human growth hormone, gene doping, blood transfusions and rHuEpo (see review [14]). It should be noted that samples are often collected for antidoping purposes at sporting or training venues after intense exercise [20] and prior intense exercise training has been reported to have significant impact on gene expression profiles of peripheral blood mononuclear cells [21] and white blood cells [22]. We previously carried out whole-blood gene expression profiling using a microarray-based approach to detect rHuEpo doping (i.e. $50 \mathrm{IU} \cdot \mathrm{kg}^{-1}$ body mass every two days for 4 weeks). Briefly, 34 of 45 selected genes from two independent groups following the microarray analysis were validated using a different quantification platform [23]. The limitations of this study were the absence of a control/placebo group and the now out-dated $\mathrm{rHuEpo}$ dosing regimen involving near clinical doses of rHuEpo as opposed to the more commonly used strategy involving rHuEpo microdosing [23]. With these limitations in mind, the aims of the present study were to investigate 1 ) whether the previously identified 45 transcriptional markers could detect $\mathrm{rHuEpo}$ microdosing in a randomised, doubleblind, placebo-controlled cross-over study; and 2) whether these gene responses differ among rHuEpo microdosing and potential confounders such as strenuous exercise training and moderate altitude exposure.

\section{Methods}

Forty-five candidate transcripts and five reference genes identified from the whole-blood transcriptome profiling in subjects administered with $50 \mathrm{IU} \cdot \mathrm{kg}^{-1}$ body mass of rHuEpo for 4 weeks (i.e. the rHuEpo high-dose study, see Additional file 1) [23] were interrogated in subjects participating in the rHuEpo microdose study and the rHuEpo confounders studies involving exercise and altitude training (Fig.1).

\section{Study design Microdose study (MDS)}

Fourteen endurance-trained healthy males (mean \pm standard deviation (SD); age: $29.9 \pm 4$ yrs., height: $178.8 \pm 4.5$ $\mathrm{cm}$, maximal aerobic capacity $\left(\dot{\mathrm{VO}}_{2} \max \right): 55.3 \pm 4.7 \mathrm{ml} \cdot \mathrm{kg}$ ${ }^{-1} \cdot \mathrm{min}^{-1}$ ) at sea-level (Glasgow, Scotland) not involved in competition during the study period participated in a randomised, double-blind, placebo-controlled crossover microdose rHuEpo study. Written informed consents were obtained from all participants. The study was approved by the University of Glasgow Ethics Committee (Scotland, UK). The subjects received $20-40 \mathrm{IU} \cdot \mathrm{kg}^{-1}$ body mass subcutaneous injections of rHuEpo (NeoRecormon, Roche, Welwyn Garden City, UK) or equivalent saline $(\mathrm{NaCl}$ 0.9\%, Baymed Healthcare Limited, Glasgow, UK; placebo injection) twice a week for 7 weeks (Fig. 2). All subjects received daily iron tablets providing approximately $105 \mathrm{mg}$ of elemental iron derived from $350 \mathrm{mg}$ of dried ferrous sulphate (Almus, Barnstaple, UK), while lactose (Minerals-Water, Purfleet, UK) substituted daily iron during the placebo trial.

All subjects were also subjected to a modified Wingate test comprising of 10 sprints of $10 \mathrm{~s}$ at baseline and during the week after the last rHuEpo or placebo injections (Fig. 2; performance data not included here). Specifically, after a 5-min cycling warm-up at $100 \mathrm{~W}$, with a flat-out sprint at $3 \mathrm{~min}$ for $5 \mathrm{~s}$, followed by a 5min rest [24], each subject performed a series of ten, maximal effort $10 \mathrm{~s}$ sprints, separated by a $50 \mathrm{~s}$ rest interval. The subject then underwent $10 \mathrm{~min}$ of active recovery at $100 \mathrm{~W}$ followed by $110 \mathrm{~min}$ of rest.

\section{Altitude training study (ATS)}

Twenty-one elite endurance runners (12 males and 9 females) were recruited (mean $\pm \mathrm{SD}$; age: $23.2 \pm 2$ yrs., height: $175.3 \pm 8.5 \mathrm{~cm}$, body mass index (BMI): $19.7 \pm$ $1.2 \mathrm{~kg} \cdot \mathrm{m}^{-2}$ ). Informed, written consents were obtained from all participants. This study was approved by the Ethics Committee of the University of Brighton (England, UK). Participants were randomly assigned into an altitude group ( $n=12$; trained at Sierra Nevada, Spain, 2320 m, for approximately $2-3$ weeks, with one participant returning from altitude after 8 days and another after 28 days)

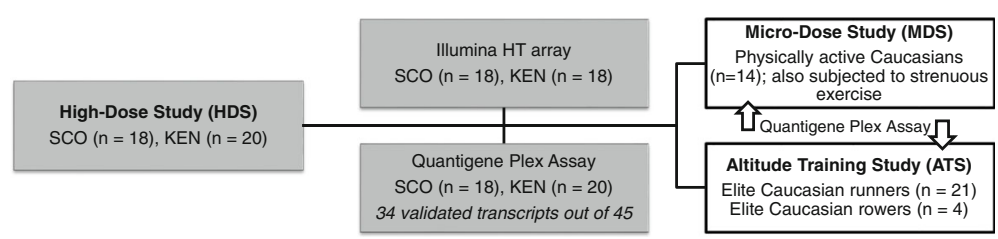

Fig. 1 A simplified diagram of the study flow specifying sample size and analytical platforms used. SCO: Scottish cohort in the HDS. KEN: Kenyan cohort in the HDS 


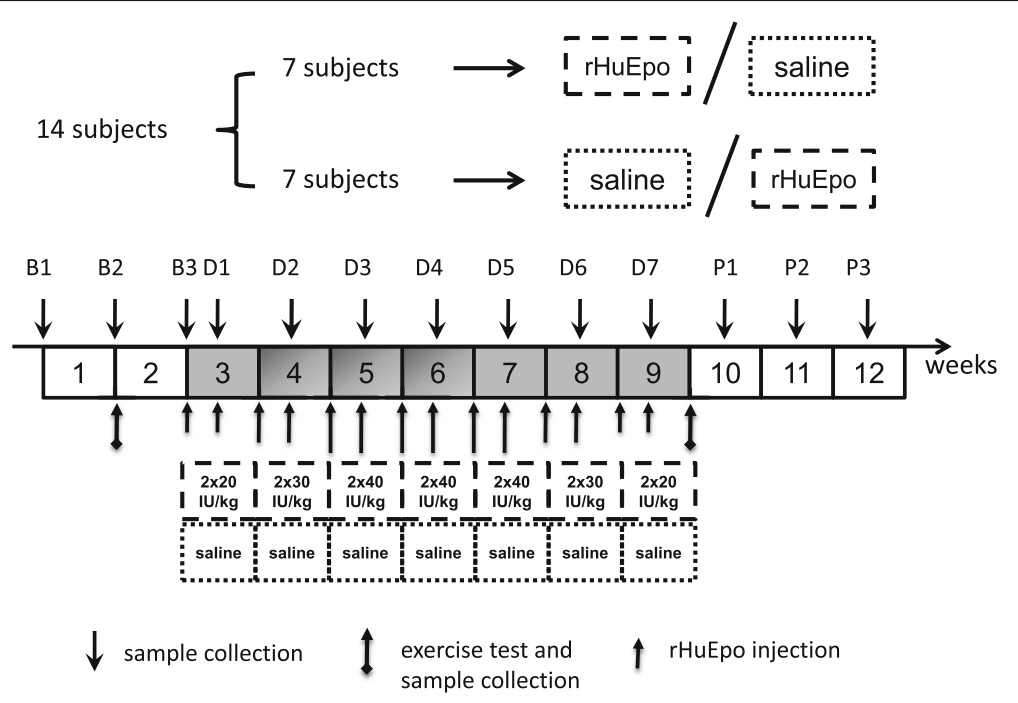

Fig. 2 MDS experimental design. B: baseline; D: during rHuEpo administration; P: post rHuEpo administration

and a control group ( $n=9$; trained at sea level for approximately 2 weeks). Both groups followed the same training programme in preparation for a major international athletics competition.

Four elite male rowers were also recruited (mean \pm SD; age: $25.3 \pm 3.6$ yrs., height: $193.3 \pm 3.6 \mathrm{~cm}$, BMI: $26.3 \pm$ $1.2 \mathrm{~kg} \cdot \mathrm{m}^{-2}$ and $\left.\dot{\mathrm{VO}}_{2} \max : 63.8 \pm 4.3 \mathrm{ml} \cdot \mathrm{kg}^{-1} \cdot \mathrm{min}^{-1}\right)$. These athletes underwent 2-week of altitude training (Santa Caterina di Valfurva, Italy, $1850 \mathrm{~m}$ ) in preparation for an international competition. Prior to and after the altitude exposure, these athletes remained in a hypoxic chamber/ altitude room $\left(16 \mathrm{~m}^{2}\right.$; excluding training and for meals) set to an oxygen concentration equivalent to $2500 \mathrm{~m}$ for 5 days. Informed and written consent was obtained from all participants and approved by the Ethics Committee of the University of Glasgow (Scotland, UK).

\section{Blood sampling and RNA extraction}

In the MDS, whole blood samples were collected in triplicate at baseline (approximately day $-14,-7$ and 0 ) and then once a week for 7 weeks during rHuEpo administration (approximately day 3, 10, 17, 24, 31, 38 and 45) and for 3 weeks post rHuEpo administration (approximately day 52, 59 and 66) (Fig. 2). Whole blood samples were also obtained before, immediately after the 10 sprints and $30 \mathrm{~min}$ after the last sprint following the modified Wingate test both at baseline and after the last rHuEpo or placebo injections (Fig. 2). In the ATS, whole blood samples were collected from the elite runners at baseline (approximately day -14 ), during (approximately day 10), 48-h-, 1-week-, 2-week- and 4-week-post altitude exposure, respectively. Samples were also obtained from the control group of elite runners at baseline (approximately day -14), 2-week- and 4-week-post the sea-level training period. Whole blood samples were collected from the elite rowers at baseline (day -14 and -10), during (day 5 and 22) and post (day 29, 33 and 39) simulated and natural altitude exposure (days are relative to the first day of the simulated altitude). For all studies, $3 \mathrm{~mL}$ whole blood sample was collected using the Tempus ${ }^{\mathrm{Tm}}$ Blood RNA tube (Life Technologies, Carlsbad, CA, USA) and mixed vigorously with $6 \mathrm{~mL}$ stabilising reagent immediately after collection. The whole sample was incubated at room temperature for approximately $3 \mathrm{~h}$ and then stored at $-20{ }^{\circ} \mathrm{C}$ before subsequent analysis. Three-millilitre whole blood was collected in $K_{3}$ EDTA tubes (Greiner Bio-One Ltd, Stonehouse, UK) for haematology analysis, and the whole blood was mixed thoroughly with the tube additive by gently inverting the tube $5-10$ times as per the manufacturer's instructions.

Total RNA was isolated from the whole blood collected using Tempus tubes according to the manufacturer's instructions (Tempus $^{\text {Tix }}$ Spin RNA Isolation Kit, Life Technologies, Carlsbad, CA, USA). The purified total RNA was eluted in $90 \mu \mathrm{L}$ elution buffer and stored in three aliquots at $-80{ }^{\circ} \mathrm{C}$ until further analysis. RNA quantity and purity was assessed by the Nanodrop ${ }^{\mathrm{TM}}$ ND-2000 Spectrophotometer (Thermo Fisher Scientific, Wilmington, DE, USA).

\section{Haematological analysis}

The mixed $\mathrm{K}_{3}$ EDTA blood tubes (Greiner Bio-One Ltd., Stonehouse, UK) was tested on the Sysmex XT-2000i (Sysmex, Norderstedt, Germany) for the MDS and ATS elite rowers samples, or the Advia 2120i system (Siemens, Worldwide) for the ATS elite runners samples. 
Standard haematological parameters were measured (i.e. haemoglobin, HGB; haematocrit, HCT; and reticulocyte percent, RET\%). These samples were measured in accordance with the WADA Athlete Biological Passport Operating Guidelines (version 4.0, 2013) [25]. The blood data was analysed using $\mathrm{R}$ lme4 (for applying the linear model) [26, 27] and phia (for post-hoc interaction analysis) [28] packages using a mixed design, with two within-subject variables (the $\mathrm{rHuEpo/placebo}$ trial and/ or time covariates) as the fixed factors and subject as the random factor, in the MDS and ATS, respectively. Significance level was adjusted using the holmbonferroni method.

\section{QuantiGene Plex experiment and data analysis}

Two hundred nanogram RNA was run in duplicate for quantification of the 45 selected RNA targets and 5 reference genes (ACTB, ACTR10, MRFAP1, PPIB and $R A B 11 A)$ in subjects participating in the MDS and ATS, using the QuantiGene Plex Assay (Affymetrix, Santa Clara, CA, USA). The resulting fluorescence signal was measured on the MAGPIX (Luminex, Austin, TX, USA). The median fluorescence intensity (MFI) values were viewed and exported from the xPonent software (Luminex, Austin, TX, USA) for statistical analysis. The MFI data was background subtracted and normalised to the geometric mean of the five reference genes. The coefficient of variation $(\mathrm{CV})$ for assay precision was calculated using the duplicated samples. The "limma" function implemented in the R limma package [29] was used to perform the differential expression analysis based on the adjusted and $\log 2$ transformed data. Gene expression values were compared to the averaged baseline or the baseline in the MDS (i.e. in the rHuEpo and placebo groups, respectively), MDS exercise samples (i.e. post vs. pre exercise as well as before-after treatment comparisons in the rHuEpo and placebo groups, respectively) and ATS (i.e. in the altitude and control groups, respectively), where appropriate. Gene expression changes over time were also examined in rHuEpo vs. placebo in the MDS and MDS exercise samples and in altitude vs. control in the elite runners. Transcripts expression exceeding a FDR [30] $<0.05$ and a 1.5 fold-change were considered meaningful in the current context.

\section{Adaptive model analysis of blood and molecular signatures}

In the MDS, the adaptive Bayesian model was applied on the two primary markers (i.e. HGB and the stimulation index OFF-score) of the haematological module of the ABP. Parameters of the model, including within- and between-subject components of variance, were chosen to represent a modal population of Caucasian male athletes aged $20-40$ yrs. [31]. The data were analysed by an investigator without knowing whether the profile was obtained from a rHuEpo or placebo sample. Following the unblinding, sensitivity was calculated based on the portion of samples in the rHuEpo group that produced at least one atypical value out of individual limits (i.e. true positives/size of the rHuEpo group), and specificity was calculated given the portion of samples in the placebo group that presented no atypical value out of individual limits (i.e. true negatives/size of placebo group). For 41 of the 45 transcripts, within- and betweensubject variances were estimated using the analysis of variance on the placebo data of $\log 2$ expression with subject as a random effect. A leave-one-out crossvalidation procedure was used to minimise overfitting. The adaptive model was applied assuming universal components of variance and normality in the withinsubject variations of the transcripts. A specificity level of 99\% was chosen for all reference ranges returned by the adaptive model. Area under the Receiving Operating Characteristic (ROC) curve was computed on the percentiles at which HGB, OFF-score and all transcript sequences were falling in the distribution of sequences as returned by the adaptive model.

\section{Results}

\section{Samples available for analysis}

In the MDS, 343 out of 364 samples (i.e. 14 subjects $\times 2$ trials $\times 13$ time points), including 174 and 169 samples following $\mathrm{rHuEpo}$ and placebo injections, respectively, were collected and analysed using the QuantiGene Plex Assay (Affymetrix, Santa Clara, CA, USA). The average CV was $10.6 \%$ across all samples analysed (vs. 15\% typical CV of the QuantiGene Plex Assay [32]). 171 (98.3\% of 174) and 163 (96.4\% of 169) samples under rHuEpo and placebo administration were available for the haematological analysis, respectively. 164 of 168 MDS exercise samples (i.e. 14 subjects $\times 2$ trials $\times 6$ exercise samples) were available for the QuantiGene Plex 2.0 Assay (Affymetrix, Santa Clara, CA, USA) analysis. The average CV was $16 \%$ across these samples. In the ATS elite runners, 66 of 72 samples (i.e. 12 subjects $\times 6$ time points) obtained following altitude training and 22 of 27 samples (i.e. 9 subjects $\times 3$ time points) obtained following sea-level training were processed using the QuantiGene Plex 2.0 Assay (Affymetrix, Santa Clara, CA, USA). 48 and 23 samples were available for HGB/ HCT analysis in the elite runners and controls, and 37 and 21 samples for RET\% calculation in the elite runners and controls, respectively. In the ATS elite rowers, 28 samples (i.e. 4 subjects $\times 7$ time points) were available for the haematological and gene expression analyses. The average CVs were $8.4 \%$ and $9.8 \%$ across the samples from elite runners and rowers, respectively. Forty-one of the 45 transcripts exceeding the assay limit of detection 
were available for gene expression analysis in the MDS and ATS and 35 for MDS exercise-induced gene expression analysis, respectively.

\section{Haematological analysis}

In the MDS, both HGB concentration and HCT percentage were gradually increased throughout the rHuEpo administration relative to the baseline values and reached the maximum one week after the last injection (Holm-Bonferroni adjusted $p<0.05)$ (Fig. 3). RET\% increased rapidly after the first injection and remained significantly elevated for 4 weeks (Holm-Bonferroni adjusted $p<0.05$ ) (Fig. 3). The RET\% was significantly lower compared to the baseline values (Holm-Bonferroni adjusted $p<0.05)$ throughout the post-rHuEpo phase (Fig. 3). The OFF-score showed an increasing trend during rHuEpo administration and significantly increased throughout the post phase (Holm-Bonferroni adjusted $p<0.05$ ) (Fig. 3). No significant differences over time were found for the HGB and HCT parameters compared to baseline values in the placebo trial in the MDS, however, RET\% were significantly increased at During 5 and Post 2 and while OFF-scores were significantly lower at During 4-7 and Post 1,2 in the placebo trial (Holm-Bonferroni adjusted $p<0.05)$. When comparing the blood data between the rHuEpo and placebo groups, similar trends and findings were obtained to those observed in the rHuEpo group. Haematological analysis in the ATS elite runners revealed a significant decrease in HGB during altitude (approximately 10 days at altitude), a significant increase in RET\% and a significant decrease in OFF-scores post 2 weeks of sea-level training (Holm-Bonferroni adjusted $p=0.036,0.02$ and 0.002 , respectively, see Additional files 2 and 3). No other significant differences were observed in these blood parameters over time at available time points (compared to the baseline), neither in the altitude group nor in the control group of the ATS elite runners (Holm-Bonferroni adjusted $p>0.05$ ). Similarly, in the ATS elite rowers, no significant changes were observed over time in comparison to baseline values for the four haematological markers (see Additional file 4).

\section{Gene expression analysis}

In the group of subjects following the rHuEpo injection in the MDS, differentially expressed genes were firstly selected based on the moderated F-statistic computed by the "eBayes" function implemented in the R limma package. Thirty-six out of the 41 genes exceeded the overall test of significance at the FDR adjusted $p$ of 0.05 . Of
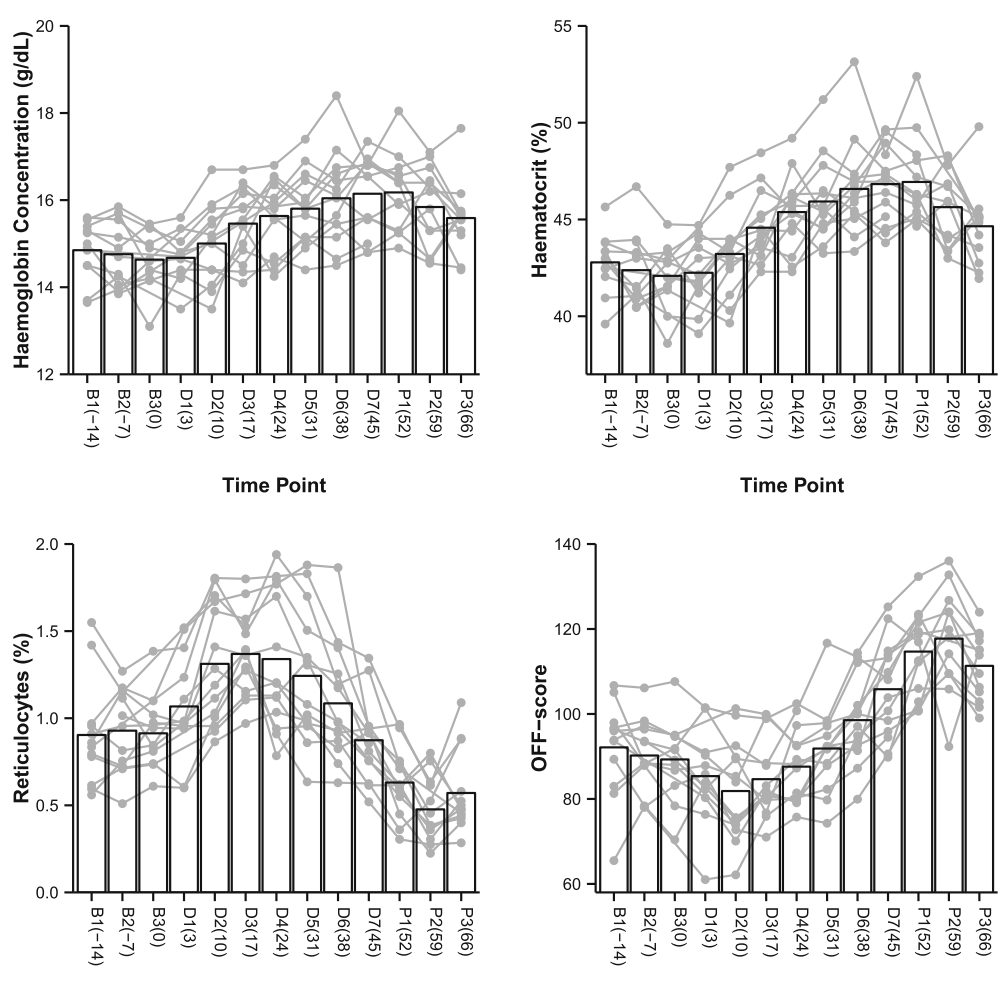

Time Point

Time Point

Fig. 3 Haemoglobin concentration, haematocrit (\%), reticulocytes (\%) and OFF-score in subjects taken rHuEpo in the MDS. Data is displayed by means with corresponding individual changes over time. B: baseline; D: during rHuEpo administration; P: post rHuEpo administration. The number in the parentheses indicates the blood sampling day relative to the day of the first rHuEpo injection (i.e. B3) 
these 36 genes, 23 were selected when individual contrasts in gene expression revealed non-zero differences seven days after the last rHuEpo injection (i.e. Post 1 ) at FDR $<0.05$. Subsequently, 17 of the 23 genes were found significantly altered in expression, exceeding a foldchange of $1.5(\mathrm{FDR}<0.05)$, ten days (i.e. During 2) after the first injection. Among the 17 genes, 11 were consistently over-expressed from During 2 (Day 10) to During 5 (Day 31) and were then under-expressed throughout the post-administration stage, i.e. Post 1 (Day 52) to Post 3 (Day 66) $(\mathrm{FDR}<0.05$ with 1.5 fold-change threshold) (Table 1). Nine of the 11 genes, ALAS2, BCL2L1, DCAF12, EPB42, GMPR, SELENBP1, SLC4A1, TMOD1 and TRIM58 (Table 1 and Additional file 5), were common with the 34 transcripts previously identified and validated [23]. None of the 41 genes were differentially expressed in the placebo group over time (FDR >0.15). When comparing the levels of gene expression in the rHuEpo group with that in the placebo group, 24 of the 41 genes were down-regulated at Post $1-3$ (FDR $<0.05$ with 1.5 fold-change threshold) (Table 2), overlapping the 9 genes aforementioned and no genes were differentially expressed in the "During" stage between the $\mathrm{rHuEpo}$ group and the placebo group (FDR $>0.17$ ).

Seven genes (1 up-, $C D 247$ and 6 down-regulated, BPGM, FECH, SNCA, STRADB, TRIM58 and YOD1) of the 35 were significantly altered immediately following 10 sprints of $10 \mathrm{~s}$ compared to baseline (i.e. pre exercise) before the rHuEpo injection and 2 genes ( 1 up-, CD247 and 1 down-regulated, LOC100130562) after the last rHuEpo injection (FDR $<0.05$ with 1.5 fold-change threshold) (see Additional file 6). One (up-regulated, $C D 247$ ) and 16 (1 up-, $C D 247$ and 15 down-regulated, ADIPOR1, BCL2L1, BPGM, CA1, DCAF12, FAM46C, FBXO7, FECH, OSBP2, SNCA, STRADB, TRIM58, $U B X N 6, Y B X 3$ and $Y O D 1)$ genes of the 35 were found significantly altered immediately after the repeated sprint tests in comparison to pre-exercise gene expression before and after the placebo injection, respectively (FDR $<$ 0.05 with 1.5 fold-change threshold) (see Additional file 6). No significant changes were found post $30 \mathrm{~min}$ of exercise vs. pre exercise in either rHuEpo or placebo trials. When

Table 111 genes differentially expressed at During 2 to 5 and throughout the post rHuEpo administration in the MDS

\begin{tabular}{|c|c|c|c|c|c|c|c|c|c|c|c|c|c|c|}
\hline Gene & $\begin{array}{l}\text { During } \\
2\end{array}$ & $\begin{array}{l}\text { During } \\
3\end{array}$ & $\begin{array}{l}\text { During } \\
4\end{array}$ & $\begin{array}{l}\text { During } \\
5\end{array}$ & Post 1 & Post 2 & Post 3 & $\begin{array}{l}\text { During } \\
2\end{array}$ & $\begin{array}{l}\text { During } \\
3\end{array}$ & $\begin{array}{l}\text { During } \\
4\end{array}$ & $\begin{array}{l}\text { During } \\
5\end{array}$ & Post 1 & Post 2 & Post 3 \\
\hline \multicolumn{8}{|l|}{ Log2 FC } & \multicolumn{7}{|l|}{ FC } \\
\hline ALAS2 & 0.86 & 0.95 & 0.80 & 0.70 & -0.82 & -1.32 & -1.18 & 1.81 & 1.93 & 1.75 & 1.63 & -1.77 & -2.50 & -2.26 \\
\hline BCL2L1 & 0.71 & 0.81 & 0.79 & 0.69 & -0.67 & -0.88 & -0.89 & 1.63 & 1.75 & 1.73 & 1.61 & -1.59 & -1.84 & -1.85 \\
\hline DCAF12 & 0.60 & 0.78 & 0.73 & 0.66 & -0.59 & -0.90 & -0.89 & 1.52 & 1.72 & 1.66 & 1.58 & -1.50 & -1.87 & -1.86 \\
\hline EPB42 & 0.86 & 0.98 & 0.88 & 0.76 & -0.89 & -1.25 & -1.08 & 1.82 & 1.97 & 1.84 & 1.69 & -1.85 & -2.37 & -2.11 \\
\hline GMPR & 0.81 & 0.94 & 0.79 & 0.68 & -0.72 & -0.94 & -0.84 & 1.75 & 1.92 & 1.73 & 1.60 & -1.65 & -1.92 & -1.79 \\
\hline OSBP2 & 0.96 & 1.08 & 1.05 & 1.00 & -0.89 & -1.16 & -1.03 & 1.95 & 2.12 & 2.07 & 2.00 & -1.85 & -2.23 & -2.04 \\
\hline SELENBP1 & 0.98 & 1.09 & 0.97 & 0.83 & -0.90 & -1.19 & -1.05 & 1.97 & 2.13 & 1.96 & 1.77 & -1.87 & -2.29 & -2.07 \\
\hline SLC4A1 & 0.89 & 1.08 & 0.96 & 0.82 & -0.87 & -1.18 & -1.10 & 1.86 & 2.12 & 1.94 & 1.77 & -1.83 & -2.26 & -2.14 \\
\hline TMOD1 & 0.65 & 0.89 & 0.76 & 0.61 & -0.71 & -1.06 & -1.00 & 1.57 & 1.85 & 1.70 & 1.53 & -1.64 & -2.08 & -2.01 \\
\hline TNS1 & 0.84 & 1.10 & 0.98 & 0.87 & -0.94 & -1.22 & -1.10 & 1.79 & 2.14 & 1.97 & 1.83 & -1.91 & -2.34 & -2.14 \\
\hline TRIM58 & 0.73 & 0.82 & 0.72 & 0.67 & -0.74 & -0.94 & -0.97 & 1.66 & 1.76 & 1.65 & 1.59 & -1.67 & -1.92 & -1.96 \\
\hline \multicolumn{8}{|c|}{ Uncorrected $P$ val. } & \multicolumn{7}{|l|}{ FDR } \\
\hline ALAS2 & 0.00181 & 0.00055 & 0.00337 & 0.01011 & 0.00274 & 0.000003 & 0.00002 & 0.00778 & 0.00173 & 0.00767 & 0.02030 & 0.01443 & 0.00006 & 0.00008 \\
\hline BCL2L1 & 0.00180 & 0.00038 & 0.00048 & 0.00230 & 0.00317 & 0.00013 & 0.00009 & 0.00778 & 0.00171 & 0.00367 & 0.01678 & 0.01443 & 0.00031 & 0.00025 \\
\hline DCAF12 & 0.00912 & 0.00072 & 0.00157 & 0.00434 & 0.01090 & 0.00014 & 0.00012 & 0.01558 & 0.00193 & 0.00430 & 0.01678 & 0.02482 & 0.00031 & 0.00031 \\
\hline EPB42 & 0.00488 & 0.00139 & 0.00416 & 0.01333 & 0.00378 & 0.00007 & 0.00045 & 0.01052 & 0.00300 & 0.00877 & 0.02277 & 0.01551 & 0.00022 & 0.00092 \\
\hline GMPR & 0.00165 & 0.00026 & 0.00200 & 0.00793 & 0.00479 & 0.00034 & 0.00110 & 0.00778 & 0.00152 & 0.00492 & 0.01835 & 0.01679 & 0.00064 & 0.00196 \\
\hline OSBP2 & 0.00024 & 0.00004 & 0.00006 & 0.00014 & 0.00071 & 0.00002 & 0.00009 & 0.00778 & 0.00079 & 0.00252 & 0.00591 & 0.01443 & 0.00008 & 0.00025 \\
\hline ELENBP1 & 0.00118 & 0.00030 & 0.00129 & 0.00603 & 0.00283 & 0.00011 & 0.00053 & 0.00778 & 0.00152 & 0.00407 & 0.01835 & 0.01443 & 0.00030 & 0.00103 \\
\hline SLC4A1 & 0.00848 & 0.00148 & 0.00478 & 0.01499 & 0.01016 & 0.00069 & 0.00126 & 0.01511 & 0.00303 & 0.00933 & 0.02458 & 0.02451 & 0.00124 & 0.00215 \\
\hline TMOD1 & 0.00520 & 0.00015 & 0.00105 & 0.00849 & 0.00218 & 0.000010 & 0.00002 & 0.01052 & 0.00152 & 0.00388 & 0.01835 & 0.01443 & 0.00008 & 0.00008 \\
\hline TNS1 & 0.00531 & 0.00026 & 0.00114 & 0.00376 & 0.00181 & 0.00007 & 0.00027 & 0.01052 & 0.00152 & 0.00388 & 0.01678 & 0.01443 & 0.00022 & 0.00058 \\
\hline TRIM58 & 0.00190 & 0.00051 & 0.00204 & 0.00450 & 0.00172 & 0.00009 & 0.00004 & 0.00778 & 0.00173 & 0.00492 & 0.01678 & 0.01443 & 0.00026 & 0.00014 \\
\hline
\end{tabular}


Table 224 genes responded differently (significantly down-regulated over Post 1, 2,3) in the rHuEpo group relative to the placebo group in the MDS

\begin{tabular}{|c|c|c|c|c|c|c|c|c|c|c|c|c|}
\hline \multirow[b]{2}{*}{ Gene } & \multicolumn{3}{|c|}{$\log 2 \mathrm{FC}$} & \multicolumn{3}{|l|}{ FC } & \multicolumn{3}{|c|}{ Uncorrected $P$ val. } & \multicolumn{3}{|l|}{ FDR } \\
\hline & Post 1 & Post 2 & Post 3 & Post 1 & Post 2 & Post 3 & Post 1 & Post 2 & Post 3 & Post 1 & Post 2 & Post 3 \\
\hline ADIPOR1 & -0.69 & -1.17 & -1.02 & -1.61 & -2.24 & -2.03 & 0.00669 & 0.00001 & 0.00007 & 0.01958 & 0.00009 & 0.00048 \\
\hline ALAS2 & -1.10 & -1.78 & -1.55 & -2.14 & -3.43 & -2.93 & 0.00504 & 0.00001 & 0.00008 & 0.01720 & 0.00009 & 0.00048 \\
\hline $\mathrm{BCL} 2 \mathrm{~L} 1$ & -0.97 & -1.29 & -1.12 & -1.96 & -2.44 & -2.18 & 0.00269 & 0.00010 & 0.00050 & 0.01720 & 0.00026 & 0.00172 \\
\hline BPGM & -0.72 & -1.11 & -1.04 & -1.64 & -2.16 & -2.05 & 0.00417 & 0.00002 & 0.00004 & 0.01720 & 0.00010 & 0.00048 \\
\hline CA1 & -0.82 & -1.28 & -1.12 & -1.76 & -2.42 & -2.18 & 0.01519 & 0.00022 & 0.00090 & 0.02967 & 0.00044 & 0.00223 \\
\hline CSDA & -0.80 & -1.25 & -1.12 & -1.75 & -2.39 & -2.18 & 0.00791 & 0.00005 & 0.00022 & 0.01959 & 0.00020 & 0.00088 \\
\hline DCAF12 & -0.77 & -1.24 & -1.05 & -1.70 & -2.36 & -2.07 & 0.01941 & 0.00024 & 0.00144 & 0.03351 & 0.00045 & 0.00282 \\
\hline EPB42 & -1.30 & -1.76 & -1.44 & -2.47 & -3.39 & -2.72 & 0.00286 & 0.00009 & 0.00097 & 0.01720 & 0.00025 & 0.00223 \\
\hline FAM46C & -0.66 & -1.12 & -0.98 & -1.58 & -2.17 & -1.98 & 0.00462 & 0.00000 & 0.00003 & 0.01720 & 0.00009 & 0.00048 \\
\hline FBXO7 & -0.64 & -1.02 & -0.94 & -1.56 & -2.03 & -1.91 & 0.00792 & 0.00003 & 0.00011 & 0.01959 & 0.00014 & 0.00049 \\
\hline $\mathrm{FECH}$ & -0.78 & -1.20 & -1.13 & -1.72 & -2.30 & -2.18 & 0.00545 & 0.00003 & 0.00007 & 0.01720 & 0.00014 & 0.00048 \\
\hline GMPR & -1.06 & -1.39 & -1.11 & -2.08 & -2.62 & -2.16 & 0.00391 & 0.00022 & 0.00249 & 0.01720 & 0.00044 & 0.00465 \\
\hline GUK1 & -0.77 & -1.26 & -1.05 & -1.71 & -2.40 & -2.07 & 0.01462 & 0.00010 & 0.00098 & 0.02967 & 0.00026 & 0.00223 \\
\hline KRT1 & -1.16 & -1.53 & -1.22 & -2.24 & -2.89 & -2.33 & 0.00181 & 0.00006 & 0.00110 & 0.01720 & 0.00021 & 0.00236 \\
\hline OSBP2 & -0.78 & -1.13 & -0.99 & -1.72 & -2.19 & -1.99 & 0.00820 & 0.00020 & 0.00086 & 0.01959 & 0.00043 & 0.00223 \\
\hline SELENBP1 & -1.28 & -1.68 & -1.39 & -2.43 & -3.21 & -2.62 & 0.00295 & 0.00014 & 0.00124 & 0.01720 & 0.00033 & 0.00254 \\
\hline SLC4A1 & -1.27 & -1.60 & -1.37 & -2.42 & -3.03 & -2.58 & 0.00860 & 0.00123 & 0.00476 & 0.01959 & 0.00209 & 0.00813 \\
\hline SNCA & -0.84 & -1.47 & -1.28 & -1.79 & -2.77 & -2.42 & 0.00952 & 0.00001 & 0.00009 & 0.02054 & 0.00009 & 0.00048 \\
\hline STRADB & -0.85 & -1.36 & -1.20 & -1.80 & -2.56 & -2.30 & 0.00438 & 0.00001 & 0.00006 & 0.01720 & 0.00009 & 0.00048 \\
\hline TMOD1 & -0.99 & -1.43 & -1.22 & -1.98 & -2.69 & -2.33 & 0.00303 & 0.00003 & 0.00025 & 0.01720 & 0.00014 & 0.00094 \\
\hline TNS1 & -1.26 & -1.63 & -1.26 & -2.40 & -3.10 & -2.39 & 0.00318 & 0.00020 & 0.00330 & 0.01720 & 0.00043 & 0.00589 \\
\hline TRIM58 & -0.93 & -1.25 & -1.15 & -1.91 & -2.38 & -2.22 & 0.00536 & 0.00027 & 0.00061 & 0.01720 & 0.00049 & 0.00181 \\
\hline UBXN6 & -0.71 & -1.23 & -1.05 & -1.64 & -2.35 & -2.07 & 0.01962 & 0.00009 & 0.00062 & 0.03351 & 0.00025 & 0.00181 \\
\hline YOD1 & -0.59 & -1.10 & -1.04 & -1.50 & -2.14 & -2.05 & 0.01831 & 0.00002 & 0.00004 & 0.03351 & 0.00011 & 0.00048 \\
\hline
\end{tabular}

Log2 FC log2 transformed fold-change, FC fold-change, FDR false discovery rate adjusted significance

comparing exercise gene expression changes before-after rHuEpo or placebo injections, no differences were observed, neither immediately nor $30 \mathrm{~min}$ post exercise $(F D R>0.93)$. Furthermore, there were no significant changes in transcription following exercise when comparing the rHuEpo group vs. the placebo group (FDR $>0.70$ ).

In the 12 elite runners, 28 out of the 41 genes exceeded an overall F-test significance at the FDR adjusted $p$ of 0.05 following altitude training (see Additional file 7 , green section). The remaining 13 genes demonstrated non-significant changes in gene expression (F-test FDR $>0.05$; see Additional file 7). Following pairwise comparisons, trends towards $5 \%$ FDR were observed for 20 genes (out of the 28) down-regulated one week after returning from altitude and 13 genes (out of the 20) were up-regulated approximately 10 days after reaching altitude (FDR $>0.059$ and 0.064, respectively; see Additional file 7). Of the 13 genes, ALAS2, BCL2L1, DCAF12, SLC4A1 TMOD1 and TRIM58 (FDR $>0.059$ with the fold-change varied from 1.39 to 1.63 , see
Additional files 7 and 8) were in common with the 9 MDS genes. In the elite runners, no genes were differentially expressed in the control group or responded differently over time in the altitude group relative to the control group post 2 and 4 weeks of altitude or sea-level training (FDR $>0.20$ and 0.60 , respectively). In the 4 elite rowers, no genes responded differently following 2-week altitude in conjunction with adaptation to simulated altitude when compared with the averaged baseline.

\section{Adaptive model analysis}

In the MDS, using 13 samples per subject collected at weekly intervals including 3 weekly samples postsupplementation, $\mathrm{rHuEpo}$ use was identified in 13 out of 14 subjects and without any false positives, for a specificity of $99 \%$ in the ABP using the haematological markers of HGB and OFF-score (Table 3); 41 out of the 45 were amenable to analysis using the adaptive model. A higher between- compared to within-subject variability was observed across the examined genes (averaged variance: 
Table 3 The adaptive model analysis summarising the withinand between-subject variances, sensitivity, specificity and ROC area for the HGB concentration, OFF-score and 41 transcripts analysed in the MDS

\begin{tabular}{|c|c|c|c|c|c|c|}
\hline & Mean & $\begin{array}{l}\text { Within- } \\
\text { subject } \\
\text { variance }\end{array}$ & $\begin{array}{l}\text { Between- } \\
\text { subject } \\
\text { variance }\end{array}$ & $\begin{array}{l}\text { Sensitivity } \\
(\%)\end{array}$ & $\begin{array}{l}\text { Specificity } \\
(\%)\end{array}$ & $\begin{array}{l}\mathrm{ROC} \\
\text { area }\end{array}$ \\
\hline $\mathrm{HGB}\left(\mathrm{g} \cdot \mathrm{L}^{-1}\right)$ & 150 & 29 & 36 & 79 & 93 & 0.87 \\
\hline OFF-score & 92 & 51 & 62 & 93 & 93 & 0.93 \\
\hline ADIPOR1 & 3.33 & 0.098 & 0.085 & 86 & 79 & 0.97 \\
\hline ALAS2 & 3.09 & 0.204 & 0.282 & 100 & 79 & 0.97 \\
\hline BCL2L1 & 1.33 & 0.159 & 0.202 & 93 & 93 & 0.96 \\
\hline BPGM & 2.74 & 0.107 & 0.087 & 93 & 71 & 0.98 \\
\hline CA1 & 1.28 & 0.159 & 0.190 & 93 & 79 & 0.97 \\
\hline CCR7 & -0.15 & 0.092 & 0.155 & 29 & 93 & 0.78 \\
\hline CD247 & -0.66 & 0.059 & 0.046 & 43 & 71 & 0.62 \\
\hline CD3D & 0.30 & 0.061 & 0.048 & 43 & 64 & 0.63 \\
\hline CSDA & 2.68 & 0.136 & 0.150 & 93 & 93 & 0.98 \\
\hline DCAF12 & 2.18 & 0.146 & 0.198 & 93 & 79 & 0.98 \\
\hline EEF1D & 1.75 & 0.026 & 0.027 & 43 & 64 & 0.63 \\
\hline EPB42 & -0.91 & 0.243 & 0.448 & 93 & 86 & 0.96 \\
\hline FAM46C & 3.74 & 0.083 & 0.061 & 93 & 79 & 0.95 \\
\hline $\mathrm{FBXO7}$ & 3.54 & 0.079 & 0.075 & 79 & 79 & 0.92 \\
\hline $\mathrm{FECH}$ & 1.34 & 0.142 & 0.119 & 86 & 71 & 0.95 \\
\hline GMPR & 0.23 & 0.181 & 0.281 & 86 & 86 & 0.93 \\
\hline GUK1 & 0.51 & 0.130 & 0.228 & 86 & 79 & 0.95 \\
\hline GYPE & -4.87 & 0.145 & 0.282 & 79 & 93 & 0.92 \\
\hline $\mathrm{HBD}$ & 0.20 & 0.243 & 0.885 & 64 & 64 & 0.95 \\
\hline KRT1 & -1.31 & 0.327 & 1.229 & 86 & 71 & 0.86 \\
\hline LEF1 & 0.37 & 0.083 & 0.129 & 36 & 93 & 0.68 \\
\hline LOC100130562 & 2.78 & 0.032 & 0.027 & 50 & 71 & 0.63 \\
\hline LOC286444 & 0.73 & 0.034 & 0.049 & 50 & 79 & 0.69 \\
\hline MIF & -0.15 & 0.043 & 0.021 & 36 & 71 & 0.62 \\
\hline OSBP2 & 0.61 & 0.205 & 0.206 & 93 & 79 & 0.97 \\
\hline PITHD1 & 0.20 & 0.125 & 0.183 & 86 & 86 & 0.92 \\
\hline RBM38 & 0.44 & 0.150 & 0.566 & 79 & 79 & 0.89 \\
\hline RNF213 & 0.76 & 0.102 & 0.062 & 36 & 79 & 0.42 \\
\hline SELENBP1 & 0.63 & 0.240 & 0.401 & 93 & 79 & 0.92 \\
\hline SGK223 & -2.81 & 0.064 & 0.042 & 36 & 79 & 0.60 \\
\hline SKAP1 & -1.52 & 0.055 & 0.052 & 43 & 79 & 0.61 \\
\hline SLC4A1 & 1.36 & 0.213 & 0.595 & 93 & 86 & 0.96 \\
\hline SNCA & 0.87 & 0.172 & 0.154 & 86 & 71 & 0.96 \\
\hline STRADB & 2.29 & 0.136 & 0.145 & 93 & 86 & 0.99 \\
\hline TMOD1 & -0.57 & 0.188 & 0.163 & 93 & 71 & 0.95 \\
\hline TNS1 & -2.05 & 0.234 & 0.306 & 93 & 86 & 0.96 \\
\hline TPRA1 & -2.43 & 0.011 & 0.010 & 50 & 79 & 0.67 \\
\hline TRIM58 & 2.23 & 0.156 & 0.199 & 86 & 71 & 0.98 \\
\hline UBXN6 & 2.06 & 0.107 & 0.209 & 93 & 71 & 0.98 \\
\hline VEGFB & -1.62 & 0.045 & 0.039 & 57 & 64 & 0.67 \\
\hline YOD1 & 1.48 & 0.137 & 0.050 & 71 & 71 & 0.90 \\
\hline
\end{tabular}

$R O C$ receiving operating characteristic
0.21 vs. 0.13 ; Table 3 ). Fifteen transcripts showed a sensitivity of $\geq 93 \%$ and a specificity of $\geq 71 \%$, with the ROC area $\geq 0.92$ (Table 3). BCL2L1 and CSDA were the genes with the highest sensitivity (93\%) and specificity (93\%), with the ROC area of 0.96 and 0.98 , respectively (Table 3). In the MDS differential gene expression analysis, the $B C L 2 L 1$ gene was significantly expressed at During $2-5$ and Post $1-3$ in the rHuEpo group (Table 1, FDR $<0.05$ and fold-change $>1.5$ ) and at Post $1-3$ in the rHuEpo group vs. the placebo group (Table 2, FDR $<$ 0.05 and fold-change $>1.5$ ); the CSDA gene was significantly down-regulated at Post $1-3$ (FDR $<0.05$ and foldchange $>1.5$ ) in the rHuEpo group vs. the placebo group (Table 2). HGB concentration $\left(\mathrm{g} \cdot \mathrm{L}^{-1}\right)$, OFF-score and gene expression changes (of the 41 transcripts) across 28 subjects (14 subjects $\times 2$ trials) obtained from the adaptive model and ROC curves are provided in Additional files 9, 10 and 11.

\section{Discussion}

Twenty-four of the 41 genes showed a significant and long lasting down-regulation following the last rHuEpo injection in the MDS given a fold-change of 1.5 and 5\% FDR spanning the post rHuEpo stage for 3 weeks (Table 2). This prolonged detection window in terms of the long lasting effect and the stability of the gene markers collected using the Tempus ${ }^{\mathrm{Tm}}$ Blood RNA method is promising and will undoubtedly improve the efficiency of rHuEpo detection given the substantially shorter detection duration of $36 \mathrm{~h}$ for improved analytical quality when using the current haematological markers [8]. Fifteen of the 24 genes showed a high sensitivity $(\geq 93 \%)$ with specificity equal to or above $71 \%$ (Table 3). Particularly, the $B C L 2 L 1$ and CSDA exhibited the highest sensitivity (93\%) and specificity (93\%) amongst the 15 genes (Table 3). The majority of the 9 genes that were consistently expressed during and post rHuEpo in the MDS rHuEpo group, namely ALAS2, BCL2L1, DCAF12, EPB42, GMPR, SELENBP1, SLC4A1, TMOD1 and TRIM58 were associated with erythrocyte membrane structure and red blood cell metabolism; these 9 genes were in common with the 34 transcripts previously identified and validated [23], while 7 of the 9 genes were in common with the 15 genes showing high sensitivity and specificity. The major spliced mRNA isoform of the ALAS2 has significant impact on erythroid heme biogenesis and hemogobin formation [33]. Erythrocyte survival is suppressed by the BH3 peptide through antagonizing $\mathrm{Bcl}-\mathrm{X}(\mathrm{L})$ [34]. EPB42 deficiency causes hereditary spherocytosis, leading to chronic haemolytic anemia with abnormally shaped erythrocytes [35]. The EPB42 may be involved in the regulation of erythrocyte shape and mechanical properties [36]. The SELENBP1 is thought to play a role in rapid cell outgrowth by determining the direction of the outgrowth and 
the synthesis of actin filaments [37]. There are two structurally and functionally distinct versions of the protein encoded by the $S L C 4 A 1$ gene - the N-terminal $40 \mathrm{kDa}$ cytoplasmic domain attaches to the red cell skeleton by binding ankyrin to maintain the structure of red blood cells and the C-terminal $50 \mathrm{kDa}$ membrane domain is responsible for the transport of anions, by facilitating the exchange of chloride and bicarbonate across the plasma membrane of erythrocytes [38-40]. The TMOD1 protein also influences the structure of the erythrocyte membrane skeleton by regulating tropomyosin [41]. The GMPR gene is mapped to chromosome 6p23 [42] and maintains the intracellular balance of guanine and adenine nucleotides [43]. It was previously reported that the human red cell glucose-6-phosphate dehydrogenase is encoded by the chromosome 6 - and chromosome $\mathrm{X}$ encoded genes [44] but subsequently disproved by other studies $[45,46]$. The DCAF12 and TRIM58 as well as CSDA genes are associated with terminal erythroid differentiation, red blood cell count and red blood cell interactome networks, respectively [47-49]. As stated previously, 5 genes (i.e. PFN1, C13orf15, TSTA3, RPL41 and TOMM40) were identified by Varlet-Marie et al. using SAGE following administration of the erythropoiesis-stimulating agent (ESA) darbepoetin alpha and high doses and microdoses of rHuEpo [19]. However, none of these genes overlapped with the molecular signature of rHuEpo doping identified and validated in our previous and current studies. Differences in study design including the specific drugs, drug administration methods, sample size and detection platforms, are all likely factors contributing to these results, emphasising further the need for rigorous replication of any markers identified in a single study.

The identification of a similar panel of genes associated with the structure and function of red blood cells identified in the present study and those of other clinical groups (see ref. [33-49]) is encouraging and should assist the development of targeted therapy to treat patients with blood disorders. There are widespread applications utilising rHuEpo in clinical settings such as the treatment of patients with anaemia of chronic renal disease, improving quality of life in cancer patients and minimising the transfusion requirement [50], but not all patients respond effectively to treatment with rHuEpo. The transcriptomic markers identified in healthy individuals administered with rHuEpo in our previous and current studies represent useful targets to investigate the signal transduction pathways activated by erythropoietin and its receptor for improved therapeutic use of ESA and rHuEpo. With reference to anti-doping, the whole-blood trancriptomic markers discovered reflect closely the RET\% changes over time (Fig. 3). This is consistent with the finding that a large amount of mRNA species in whole blood originate from reticulocytes, evidenced by the separate clustering of whole blood samples treated with RNase $\mathrm{H}$ and leukocyte samples in microarray analysis [51]. New approaches that are able to interrogate the whole transcriptome with improved dynamic range for adequate quantification (e.g. RNA-seq) and preferably in leucocytes populations in a sufficient number of participants are warranted in future anti-doping transcriptomic studies and especially those involving the manipulation of red blood cells to improve further the detection of blood doping using molecular markers. Approaches enabling the interrogations of whole genome, transcriptome and metabolome have the increased capacity to measure rapidly and in the near future, inexpensively a large number of molecular signatures, which will collectively aid the decision making during identifying and differentiating numerous doping substances and methods by ABP experts when reviewing passports. For example, one of the transcripts validated in the present study is the BPGM gene (Table 2), encoding the 2-3 BPG. It is well known that blood doping can affect 2-3 BPG metabolism and is therefore closely monitored by the WADA ABP Expert Panel. In addition, the only subject evading detection by the haematological model of ABP in the present study (see Additional files 9 and 10, subject $\mathrm{R}$ ) had a low mean cell volume and mean cell haemoglobin, indicating iron deficiency or a defect in iron metabolism and particularly that the subject participated in the present study is non-responsive to iron supplementation. However, 25 of the 41 genes included in the adaptive model analysis identified rHuEpo use in the same subject (see Additional file 11, subject 9). This illustrates the need for a holistic approach to drug detection; one based on the inclusion of a variety of parameters that provide information on related pathways and metabolism (e.g. iron, transferring and total iron binding capacity). Notably, the large-scale omics studies combined with the ABP are anticipated to be able to dramatically improve doping detection. It is also important to note that the potential for the validated transcripts in the present study to identify the rHuEpo use is high both when the transcripts are used alone (Tables 1 and 2; 24 genes exceeding a fold-change of 1.5 at 5\% FDR post $\mathrm{rHuEpo}$ injections) or in combination with the current ABP (Table 3; 20 genes with a ROC area > 0.95), emphasising the need to understand erythropoiesis using a systems biology approach.

The $C D 247$ gene was significantly up-regulated exceeding a fold-change of 1.5 at 5\% FDR immediately following exercise when compared to the baseline before and after $\mathrm{rHuEpo/placebo,} \mathrm{respectively;} \mathrm{but} \mathrm{this} \mathrm{response}$ rapidly subsided $30 \mathrm{~min}$ after exercise. The CD247 encodes the T-cell receptor zeta, which is a component of the T-cell receptor-CD3 complex [52]. Low expression 
of the gene may relate to impaired immune response $[53,54]$. This current observation is in line with previous studies indicating acute inflammatory response following acute resistance exercise $[55,56]$ and the subsequent anti-inflammatory response is to prevent the development of chronic inflammation [21, 57]. Six and fifteen genes were significantly down-regulated (6 is included in the 15) immediately following exercise before rHuEpo and after placebo, respectively (FDR $<0.05$ and foldchange $>1.5$ ). Among these genes, only TRIM58 overlaps the 9 MDS genes. TRIM58 specifically expresses during late erythroid maturation, coinciding with enucleation and dynein loss [58]. The suppression of TRIM58 relates to reduced enucleation [58]. TRIM58 gene polymorphisms associate with the circulating erythrocyte size and number [48, 59]. Previous studies reported an increased number of leukocytes immediately following heavy exercise $[21,22]$, whereas the reduced level of TRIM58 following the repeated sprint test in the present study may reflect reduced proportions of reticulocytes in accordance with previous research [58]. This modified Wingate test did not reveal significant gene expression changes using the 35 transcripts when comparing before with after $\mathrm{rHuEpo/placebo,} \mathrm{or} \mathrm{rHuEpo} \mathrm{vs.} \mathrm{placebo.}$ These results, although preliminary, argue against exercise/training being a confounder as all gene expression alterations post strenuous exercise were restored after $30 \mathrm{~min}$ and well within the two hours stipulated by WADA before a blood sample can be obtained for antidoping purposes [60]. Nevertheless, the explicit role of exercise needs to be further investigated not only in whole blood but also peripheral blood cells in order to better understand the molecular adaptations to both acute and chronic exercise (i.e. exercise training) and avoid confounding the analysis.

Previous studies have shown that short or prolonged residency at high altitude stimulates the secretion of Epo from the kidney [61-64]. For example, elevated levels of Epo are detected as early as $8 \mathrm{~h}$ after arrival at high altitude and reaches a peak $24 \mathrm{~h}$ after arrival [61]. These higher levels of Epo are also maintained throughout a period of high altitude exposure (e.g., ranging from 11 days to 4 weeks at approximately $3500 \mathrm{~m}$ or above $[61,64])$. In the present ATS, 13 out of the 41 genes failed to show statistically significant changes at the transcriptional level following approximately 2-week training at moderate altitude in 12 elite runners given a F-test FDR $>0.05$. Six of the remaining 28 genes that tended to be stimulated by altitude were in common with the 9 MDS genes (FDR $>0.059$ with the foldchange varied from 1.39 to 1.63 during and after one week of altitude training; see Additional files 7 and 8). Among these 6 genes, SLC4A1 showed a tendency towards a 1.63-fold down-regulation $(\mathrm{FDR}=\sim 0.059)$ one week after altitude training and TMOD1 a tendency for a 1.50-fold up-regulation (FDR $=\sim 0.064$ ) after ten days of altitude exposure; none of the other genes exceeded the 1.5 fold-change cut-off (FDR $>0.059$ ) (see Additional file 7). In comparison, in the MDS rHuEpo group, DCAF12 showed a least 1.52-fold up-regulation $(F D R=\sim$ 0.016 ) ten days after the first $\mathrm{rHuEpo}$ injection (i.e. During 2) and this level of expression was maintained for 4 weeks for all 6 genes (i.e. During 2-5, FDR $<0.05$, Table 1). In addition, there was at least 1.50 -fold downregulation (FDR $=\sim 0.025)$ in DCAF12 one week after the last rHuEpo injection (i.e. Post 1) with this level of change lasting throughout the 3 weeks for all 6 genes (i.e. Post $1-3, \mathrm{FDR}<0.05$, Table 1 ), with majority of the 6 genes approaching or exceeding a 2 fold-change at the post rHuEpo stage (Table 1). There was also a more pronounced gene expression response in these 6 genes post rHuEpo administration compared to the placebo group (FDR $<0.05$, Table 2), with an approximate 2.5 foldchange in ALAS2, SLC4A1 and TMOD1 2 and 3 weeks after the last injections (i.e. Post 2, 3, Table 2). As stated previously, the observed patterns of change in gene expression in MDS reflect closely the RET\% changes over time (Fig. 3). In comparison, haematological analysis in the ATS elite runners revealed a small but significant decrease in HGB during altitude (approximately 10 days at altitude) (FDR $=0.036$, see Additional file 2). No genes were differentially expressed in the control group or responded differently over time in the altitude group relative to the control group and this trend was in general agreement with the haematological results that showed no change over time despite exposure to altitude. The relatively long lasting effects and high magnitude of changes of the validated transcriptional markers of rHuEpo compared with the gene expression changes of the same markers (i.e. the 6 genes) in the ATS elite runners provide strong evidence in favour of applying such markers, alongside current anti-doping strategies to detect blood doping. The finding of no change in gene expression or in the measured haematological variables in the four elite rowers most likely reflects the limited exposure to moderate altitude, the small sample size and the typical inter-individual variation (see additional file 4). An altitude study with extended exposure (for 4 weeks at least) at high altitude $(\sim 3000 \mathrm{~m})$ involving the analysis of both whole blood and peripheral blood for omics marker identification is required to better understand the molecular adaptations to altitude as compared to blood doping. Only then can the molecular response to altitude training be excluded, or if needed, integrated in the ABP along with other confounders to detect blood doping. Assessing and determining other confounding variables influencing the biological and analytical variability of the gene markers through altered erythropoiesis presents a critical next step 
to enhance the specificity and sensitivity of current $\mathrm{ABP}$ for unbiased detection of blood doping in conjunction with a systems biology approach.

\section{Conclusions}

In conclusion, several human whole-blood transcriptional signatures signifying predominantly altered red blood cell production were identified following rHuEpo injections ranging from high doses to microdoses. These findings support the use of molecular markers as potential biomarkers with an improved detection window and high sensitivity and specificity for developing the transcriptionallyenhanced ABP model for detecting blood doping. Collectively, the findings of the present study, interpreted in the context of the latest omics research, are encouraging and suggest a systems biology approach combining various omics signatures from genomics, transcriptomics, proteomics and metabolomics will inevitably provide a deeper understanding of the effects of erythropoietic stimulating agents on erythropoiesis with unparalleled potential to improve current drug detection strategies with particular reference to blood doping. However, continuous and rigorous efforts will be required to determine, accommodate and possibly eliminate other confounding effects on blood doping.

\section{Additional files}

Additional file 1: List of the 50 genes for the QuantiGene Plex Assay analysis in the MDS and ATS. (DOC $67 \mathrm{~kb}$ )

Additional file 2: Haemoglobin concentration $\left(\mathrm{g} \cdot \mathrm{dL}^{-1}\right)$, haematocrit $(\%)$, reticulocytes (\%) and OFF-score in response to altitude training in the 11 ATS elite runners. Data is displayed by means with corresponding individual changes over time. $B(-7), D(10), P(16), P(30)$ and $\mathrm{P}(42)$ represent pre, during, 48-h-, 1-week-, and 4-week-post altitude exposure, respectively. (PDF $7 \mathrm{~kb}$ )

Additional file 3: Haemoglobin concentration ( $\left.g \cdot d L^{-1}\right)$, haematocrit (\%), reticulocytes (\%) and OFF-score changes in the 7 ATS elite runners at the sea level. Data is displayed by means with corresponding individual changes over time. $B(-7), P(30)$ and $P(42)$ represent pre, 1-week-, and 4-week-post altitude exposure, respectively. (PDF $6 \mathrm{~kb}$ )

Additional file 4: Haemoglobin concentration $\left(\mathrm{g} \cdot \mathrm{dL}^{-1}\right)$, haematocrit (\%), reticulocytes (\%) and OFF-score in the four ATS elite rowers. Data is displayed by means with corresponding individual changes over time. B1 (-14) and B2(-10): 14 and 10 days prior to 5-day simulated altitude, respectively; D1 (5), D2(22), P1(29), P2(33) and P3(39): 5, 22, 29, 33 and 39 days relative to the first day of the simulated altitude prior to the natural altitude exposure. (PDF $7 \mathrm{~kb}$ )

Additional file 5: Individual expression of the ALAS2, BCL2L1, DCAF12, EPB42, GMPR, SELENBP1, SLC4A1, TMOD1 and TRIM58 genes over time in response to rHuEpo in the MDS, respectively. (PDF $23 \mathrm{~kb}$ )

Additional file 6: Genes differentially expressed immediately following the repeated sprint tests vs. baseline prior to and after the rHuEpo/ placebo injection in the MDS, respectively. (XLS $21 \mathrm{~kb}$ )

Additional file 7: Summary of gene expression changes over time in response to altitude exposure in the 12 ATS elite runners for the 41 transcripts. (XLS $37 \mathrm{~kb}$ )

Additional file 8: Individual expression of the ALAS2, BCL2L1, DCAF12, SLC4A1,TMOD1 and TRIM58 genes over time in response to altitude exposure in the 12 ATS elite runners, respectively. $B(-7), D(10), P(16)$, $P(21), P(30)$ and $P(42)$ represent pre, during, 48-h-, 1-week-, 2-week- and 4-week-post altitude exposure, respectively. (PDF $9 \mathrm{~kb}$ )

Additional file 9: Adaptive model analysis and ROC curve in HGB concentration $\left(\mathrm{g} \cdot \mathrm{L}^{-1}\right)$ across 28 subjects ( 14 subjects $\times 2$ trials) in the MDS. Red lines: individual limits as determined by the adaptive model for a specificity of 99\%; blue line: actual test results. Subject A, D, F. G, J, K, M, $P, R, S, U, X, Z$ and $A A$ participated in the rHuEpo trial; subject $B, C, E, H, I$, $L, N, O, Q, T, V, W, Y$ and $B B$ participated in the placebo trial. X-axis: 13 time points and $\mathrm{y}$-axis: $\mathrm{HGB}$ concentrations. (ZIP $88 \mathrm{~kb}$ )

Additional file 10: Adaptive model analysis and ROC curve in OFF-score across 28 subjects ( 14 subjects $\times 2$ trials) in the MDS. Red lines: individual limits as determined by the adaptive model for a specificity of $99 \%$; blue line: actual test results. Subject $A, D, F . G, J, K, M, P, R, S, U, X, Z$ and $A A$ participated in the rHuEpo trial; subject B, C, E, H, I, L, N, O, Q, T, V, W, X and $A B$ participated in the placebo trial. $X$-axis: 13 time points and $y$-axis: OFF-scores. (ZIP $87 \mathrm{~kb}$ )

Additional file 11: Adaptive model analysis and ROC curve in gene expression changes of the 41 transcripts across 28 subjects (14 subjects $\times 2$ trials) in the MDS. Red lines: individual limits as determined by the adaptive model for a specificity of $99 \%$; blue line: actual test results. The first 1-14 graphs: subjects participated in the rHuEpo trial and following 15-28 graphs: the same subjects participated in the placebo trial. X-axis: 13 time points and y-axis: log2 gene expression. (ZIP $1076 \mathrm{~kb}$ )

\section{Acknowledgements}

The authors would like to thank all volunteers for their participation and cooperation as well as Mr. Mark Lees and Dr. Jun Wang for their help with data collection in the MDS.

Funding

The research and publication costs were funded by grants from the WADA (12C09YP and 13C28YP) and Affymetrix/Panomics.

\section{Availability of data and materials}

All data generated or analysed during this study are included in this published article and the additional files.

\section{About this supplement}

This article has been published as part of BMC Genomics Volume 18 Supplement 8, 2017: Proceedings of the 34th FIMS World Sports Medicine Congress. The full contents of the supplement are available online at https://bmcgenomics.biomedcentral.com/articles/supplements/ volume-18-supplement-8.

\section{Authors' contributions}

YPP and JD study design; JD, MM and AK data collection; AM medical supervision; GW, BY, JS, NF, and MM molecular lab analysis; GW, PES, BY, JS and JDM statistical analysis; GW, PES, JS and YPP data interpretation; GW and PES illustration generation; GW, PES, JS and YPP drafting the manuscript; GW and YPP manuscript revision; all authors to comment, further edit and approve the manuscript.

\section{Ethics approval and consent to participate}

Written informed consents were obtained from all subjects participated in the MDS and ATS. The studies presented were approved by the University of Glasgow Ethics Committee (Scotland, UK) for MDS and ATS elite rowers and the Ethics Committee of the University of Brighton (England, UK) for ATS elite runners.

\section{Consent for publication}

Written informed consent was obtained from the individuals involved in the study.

\section{Competing interests}

The authors declare that they have no competing interests. 


\section{Publisher's Note}

Springer Nature remains neutral with regard to jurisdictional claims in published maps and institutional affiliations.

\begin{abstract}
Author details
'Centre of Sports Medicine for Anti-Doping Research, University of Brighton, Eastbourne, UK. ${ }^{2}$ Institute of Cardiovascular and Medical Sciences, College of Medical, Veterinary and Life Sciences, University of Glasgow, Glasgow, UK. ${ }^{3}$ Brighton and Sussex Medical School, Brighton, UK. ${ }^{4}$ Faculty of Medicine, University of Tartu, Tartu, Estonia. ${ }^{5}$ Graduate School of Health and Sports Science, Juntendo University, Chiba, Japan. ${ }^{6}$ School of Sport, Health and Applied Science, St Mary's University, Twickenham, London, UK. ${ }^{7}$ Centre for Sports and Exercise, University of Edinburgh, Edinburgh, UK. ${ }^{8}$ Affymetrix, Santa Clara, CA, USA. 'BioKaizen Lab SA, Monthey, Switzerland. ${ }^{10}$ Department of Movement, Human and Health Sciences, University of Rome "Foro Italico", Rome, Italy.
\end{abstract}

\section{Published: 14 November 2017}

\section{References}

1. Sottas PE, Robinson N, Rabin O, Saugy M. The athlete biological passport. Clin Chem. 2011;57:969-76.

2. Lasne F, de Ceaurriz J. Recombinant erythropoietin in urine. Nature. 2000; 405:635.

3. Lundby C, Achman-Andersen NJ, Thomsen JJ, Norgaard AM, Robach P. Testing for recombinant human erythropoietin in urine: problems associated with current anti-doping testing. J Appl Physiol. 2008;105:417-9.

4. Schwenke D. Improved detection of EPO in blood and urine based on novel velum SAR precast horizontal gels optimized for routine analysis. 2015; http://www.dyeagnostics.com/site/wp-content/uploads/2015/02/ Application_Note_Improvements-for-EPO-detection_Schwenke_2015.pdf. Accessed 22 Jan 2017.

5. Harmonization of Analysis and Reporting of Erythropoiesis-Stimulating Agents (ESAs) by Electrophoretic Techniques. 2014; https://www.wada-ama. org/sites/default/files/resources/files/WADA-TD2014EPO-SummaryModifications-EN.PDF. Accessed 22 Jan 2017.

6. Parisotto R, Gore CJ, Emslie KR, Ashenden MJ, Brugnara C, Howe C, et al. A novel method utilising markers of altered erythropoiesis for the detection of recombinant human erythropoietin abuse in athletes. Haematologica. 2000; 85:564-72.

7. Sottas PE, Robinson N, Saugy M. The athlete's biological passport and indirect markers of blood doping. Handb Exp Pharmacol. 2010;195:305-26.

8. Lombardi G, Lanteri P, Colombini A, Lippi G, Banfi G. Stability of haematological parameters and its relevance on the athlete's biological passport model. Sports Med. 2011;41:1033-42.

9. Lippi G, Plebani M. Athlete's biological passport: to test or not to test? Clin Chem Lab Med. 2011:49:1393-5.

10. Sanchis-Gomar F, Martinez-Bello VE, Gomez-Cabrera MC, Viña J. Current limitations of the Athlete's biological passport use in sports. Clin Chem Lab Med. 2011;49:1413-5.

11. Ashenden M, Gough CE, Garnham A, Gore CJ, Sharpe K. Current markers of the athlete blood passport do not flag microdose EPO doping. Eur J Appl Physiol. 2011;111:2307-14.

12. Grover RF, Weil JV, Reeves JT. Cardiovascular adaptation to exercise at high altitude. Exerc Sport Sci Rev. 1986;14:269-302.

13. Mairbäurl H. Red blood cells in sports: effects of exercise and training on oxygen supply by red blood cells. Front Physiol. 2013;4:332.

14. Reichel C. OMICS-strategies and methods in the fight against doping. Forensic Sci Int. 2011;213:20-34

15. Pitsiladis YP, Durussel J, Rabin O. An integrative 'omics' solution to the detection of recombinant human erythropoietin and blood doping. $\mathrm{Br}$. Sports Med. 2014;48:856-61.

16. Naukkarinen J, Rissanen A, Kaprio J, Pietiläinen KH. Causes and consequences of obesity: the contribution of recent twin studies. Int J Obes. 2012:36:1017-24.

17. McCarthy MI. Genomics, type 2 diabetes, and obesity. N Engl J Med. 2010; 363:2339-50.

18. Vucic EA, Thu KL, Robison K, Rybaczyk LA, Chari R, Alvarez CE, et al. Translating cancer 'omics' to improved outcomes. Genome Res. 2012;22: $188-95$.
19. Varlet-Marie E, Audran M, Ashenden M, Sicart MT, Piquemal D. Modification of gene expression : help to detect doping with erythropoiesis-stimulating agents. Am J Hematol. 2009;84:755-9.

20. Rupert JL. Transcriptional profiling: a potential anti-doping strategy. Scand J Med Sci Sports. 2009;19:753-63.

21. Connolly PH, Caiozzo VJ, Zaldivar F, Nemet D, Larson J, Hung SP, et al. Effects of exercise on gene expression in human peripheral blood mononuclear cells. J Appl Physiol (1985). 2004;97:1461-9.

22. Büttner $P$, Mosig $S$, Lechtermann $A$, Funke $H$, Mooren FC. Exercise affects the gene expression profiles of human white blood cells. J Appl Physiol (1985). 2007;102:26-36.

23. Durussel J, Haile DW, Mooses K, Daskalaki E, Beattie W, Mooses M, et al. The blood transcriptional signature of recombinant human erythropoietin administration and implications for anti-doping strategies. Physiol Genomics. 2016:48:202-9.

24. Davison RC, Wooles AL. Cycling. In: Winter EM, Jones AM, Davison RC, Bromley PD, Mercer TH, editors. Sport and exercise physiology testing guidelines: the British Association of Sport and Exercise Sciences Guide. Oxon: Routledge; 2006. p. 364

25. WADA Athlete Biological Passport operating guidelines \& compilation of required elements. https://www.wada-ama.org/sites/default/files/ resources/files/WADA-ABPOperating-Guidelines_v4.0-EN.pdf. Accessed 16 Oct 2017.

26. Bates D, Maechler M, Bolker B, Walker S. Fitting Linear Mixed-Effects Models Using Ime4. J Stat Softw. 2015;67:1-48.

27. Bates $D$, Maechler M, Bolker B, Walker S. Fitting linear mixed-effects models using Ime4. J Stat Softw. 2015;67:1-48.

28. De Rosario-Martinez H. Phia: post-hoc interaction analysis. R package version 0.2-0. 2015. http://CRAN.R-project.org/package=phia. Accessed 16 Oct 2017.

29. Smyth GK. Linear models and empirical bayes methods for assessing differential expression in microarray experiments. Stat Appl Genet Mol Biol. 2004;3:Article3.

30. Benjamini $Y$, Hochberg $Y$. Controlling the false discovery rate: a practical and powerful approach to multiple testing. J R Statist Soc B. 1995;57:289-300.

31. Sottas PE, Robinson N, Saugy M. A forensic approach to the interpretation of blood doping markers. Law Probability \& Risk. 2008;7:191-210.

32. QuantiGene ${ }^{\oplus}$ Plex Assay. https://tools.thermofisher.com/content/sfs/ brochures/quantigene-assaysapplication-guide.pdf. Accessed 22 Jan 2017.

33. Cox TC, Sadlon TJ, Schwarz QP, Matthews CS, Wise PD, Cox LL, et al. The major splice variant of human 5 -aminolevulinate synthase-2 contributes significantly to erythroid heme biosynthesis. Int J Biochem Cell Biol. 2004;36: 281-95.

34. Walsh M, Lutz RJ, Cotter TG, O'Connor R. Erythrocyte survival is promoted by plasma and suppressed by a Bak-derived BH3 peptide that interacts with membrane-associated Bcl-X(L). Blood. 2002;99:3439-48.

35. Kalfa TA, Connor JA, Begtrup AH. EPB42-related hereditary Spherocytosis. In: AM PRA, Ardinger HH, et al., editors. GeneReviews ${ }^{\oplus}$. Seattle: University of Washington, Seattle; 2014.

36. Dahl KN, Parthasarathy R, Westhoff CM, Layton DM, Discher DE. Protein 4.2 is critical to CD47-membrane skeleton attachment in human red cells. Blood. 2004;103:1131-6.

37. Miyaguchi K. Localization of selenium-binding protein at the tips of rapidly extending protrusions. Histochem Cell Biol. 2004;121:371-6.

38. Low PS. Structure and function of the cytoplasmic domain of band 3: center of erythrocyte membrane-peripheral protein interactions. Biochim Biophys Acta. 1986;864:145-67.

39. Low PS, Zhang D, Bolin JT. Localization of mutations leading to altered cell shape and anion transport in the crystal structure of the Cytoplasmic domain of band 3. Blood Cells Mol Dis. 2001;27:81-4.

40. Reithmeier RA, Casey JR, Kalli AC, Sansom MS, Alguel Y, Iwata S. Band 3, the human red cell chloride/bicarbonate anion exchanger (AE1, SLC4A1), in a structural context. Biochim Biophys Acta. 2016;1858:1507-32.

41. Yao W, Sung LA. Erythrocyte tropomodulin isoforms with and without the N-terminal actin-binding domain. J Bio Chem. 2010;285:31408-17.

42. Murano I, Tsukahara M, Kajii T, Yoshida A. Mapping of the human guanosine monophosphate reductase gene (GMPR) to chromosome 6 p23 by fluorescence in situ hybridization. Genomics. 1994;19:179-80.

43. Hedstrom $L$. The dynamic determinants of reaction specificity in the IMPDH/GMPR family of (B/a)8 barrel enzymes. Crit Rev Biochem Mol Biol. 2012;47:250-63 
44. Kanno H, Huang IY, Kan YW, Yoshida A. Two structural genes on different chromosomes are required for encoding the major subunit of human red cell glucose-6-phosphate dehydrogenase. Cell. 1989;58:595-606.

45. Beutler E, Gelbart T, Kuhl W. Human red cell glucose-6-phosphate dehydrogenase: all active enzyme has sequence predicted by the $X$ chromosome-encoded cDNA. Cell. 1990;62:7-9.

46. Mason PJ, Bautista JM, Vulliamy TJ, Turner N, Luzzatto L. Human red cell glucose-6-phosphate dehydrogenase is encoded only on the X chromosome. Cell. 1990;62:9-10.

47. An X, Schulz VP, Li J, Wu K, Liu J, Xue F, et al. Global transcriptome analyses of human and murine terminal erythroid differentiation. Blood. 2014;123: 3466-77.

48. van der Harst P, Zhang W, Mateo Leach I, Rendon A, Verweij N, Sehmi J, et al. Seventy-five genetic loci influencing the human red blood cell. Nature. 2012;492:369-75.

49. D'Alessandro A, Righetti PG, Zolla L. The red blood cell proteome and Interactome: an update. J Proteome Res. 2010;9:144-63.

50. Ng T, Marx G, Littlewood T, Macdougall I. Recombinant erythropoietin in clinical practice. Postgrad Med J. 2003;79:367-76.

51. Feezor RJ, Baker HV, Mindrinos M, Hayden D, Tannahill CL, Brownstein BH, et al. Whole blood and leukocyte RNA isolation for gene expression analyses. Physiol Genomics. 2004;19:247-54.

52. Clevers H, Alarcon B, Wileman T, Terhorst C. The T cell receptor/CD3 complex: a dynamic protein ensemble. Annu Rev Immunol. 1988;6:629-62.

53. Christopoulos P, Dopfer EP, Malkovsky M, Esser PR, Schaefer HE, Marx A, et al. A novel thymoma-associated immunodeficiency with increased naive T cells and reduced CD247 expression. J Immunol. 2015;194:3045-53.

54. Bronstein-Sitton N, Cohen-Daniel L, Vaknin I, Ezernitchi AV, Leshem B, Halabi A, et al. Sustained exposure to bacterial antigen induces interferon-gammadependent T cell receptor zeta down-regulation and impaired T cell function. Nat Immunol. 2003;4:957-64.

55. Carlson LA, Tighe SW, Kenefick RW, Dragon J, Westcott NW, Leclair RJ. Changes in transcriptional output of human peripheral blood mononuclear cells following resistance exercise. Eur J Appl Physiol. 2011;111:2919-29.

56. Storey AG, Birch NP, Fan V, Smith HK. Stress responses to short-term intensified and reduced training in competitive weightlifters. Scand J Med Sci Sports. 2016;26:29-40

57. Gjevestad GO, Holven KB, Ulven SM. Effects of exercise on gene expression of inflammatory markers in human peripheral blood cells: a systematic review. Curr Cardiovasc Risk Rep 2015;9:34.

58. Thom CS, Traxler EA, Khandros E, Nickas JM, Zhou OY, Lazarus JE, et al. Trim58 degrades Dynein and regulates terminal erythropoiesis. Dev Cell. 2014:30:688-700.

59. Kamatani Y, Matsuda K, Okada Y, Kubo M, Hosono N, Daigo Y, et al. Genome-wide association study of hematological and biochemical traits in a Japanese population. Nat Genet. 2010;42:210-5.

60. Athlete Biological Passport Operating Guidelines \& Compilation of Required Elements. 2014; https://www.wada-ama.org/sites/default/files/resources/ files/wada_abp_operating_guidelines_2014_v5.0_en.pdf. Accessed 23 Jan 2017.

61. Basu M, Malhotra AS, Pal K, Prasad R, Kumar R, Prasad BA, et al. Erythropoietin levels in lowlanders and high-altitude natives at $3450 \mathrm{~m}$. Aviat Space Environ Med. 2007;78:963-7.

62. Gunga HC, Röcker L, Behn C, Hildebrandt W, Koralewski E, Rich I, et al. Shift working in the Chilean Andes (> 3,600 $\mathrm{m}$ ) and its influence on erythropoietin and the low-pressure system. J Appl Physiol (1985). 1996;81: $846-52$

63. Hudson JG, Bowen AL, Navia P, Rios-Dalenz J, Pollard AJ, Williams D, et al. The effect of high altitude on platelet counts, thrombopoietin and erythropoietin levels in young Bolivian airmen visiting the Andes. Int J Biometeorol. 1999:43:85-90.

64. Milledge JS, Cotes PM. Serum erythropoietin in humans at high altitude and its relation to plasma renin. J Appl Physiol (1985). 1985;59:360-4.

\section{Submit your next manuscript to BioMed Central and we will help you at every step:}

- We accept pre-submission inquiries

- Our selector tool helps you to find the most relevant journal

- We provide round the clock customer support

- Convenient online submission

- Thorough peer review

- Inclusion in PubMed and all major indexing services

- Maximum visibility for your research

Submit your manuscript at www.biomedcentral.com/submit
Biomed Central 